\title{
Stent retriever thrombectomy combined with local thrombolytic therapy for cerebral venous sinus thrombosis: A case report
}

\author{
CHENGWEI CHEN ${ }^{1,2^{*}}$, QIUJING WANG ${ }^{2,3^{*}}$, XIFENG LI $^{2 *}$, ZIMING LU $^{2}$, JIAN HE$^{2}$, \\ QINRUI FANG ${ }^{2}$, XUNCHANG KE ${ }^{2}, \mathrm{CHUANZHI} \mathrm{DUAN}^{2}$ and TIELIN LI ${ }^{2}$
}

\begin{abstract}
${ }^{1}$ Department of Neurosurgery, The First Affiliated Hospital of Zhejiang Chinese Medical University, Hangzhou, Zhejiang 310006; ${ }^{2}$ The National Key Clinical Specialty, The Engineering Technology Research Center of Education Ministry of China, Guangdong Provincial Key Laboratory on Brain Function Repair and Regeneration, Department of Neurosurgery, Zhujiang Hospital, Southern Medical University, Guangzhou, Guangdong 510282; ${ }^{3}$ Department of

Neurosurgery, Shenzhen Hospital of Southern Medical University, Shenzhen, Guangdong 518000, P.R. China
\end{abstract}

Received January 1, 2016; Accepted February 1, 2017

DOI: $10.3892 /$ etm.2017.5043

\begin{abstract}
The present study reports the effect of successful treatment of cerebral venous sinus thrombosis (CVST) with stent retriever thrombectomy combined with local thrombolytic therapy. Medical records of 29 patients were retrospectively analyzed following a diagnosis of CVST with magnetic resonance venography (MRV) or digital subtraction angiography (DSA). Systemic anticoagulation was the initial treatment in all patients following admission. In group A, stent retriever thrombectomy combined with local thrombolytic therapy was performed on 14 patients who met the criteria of endovascular therapy. Stent-assisted angioplasty was also performed when patients presented with venous sinus stenosis. A total of 15 patients in group B received systemic anticoagulant treatment. Subsequently, warfarin was administered orally for 3 to 12 months as a continuous anticoagulant therapy. International normalized ratio was monitored when patients were receiving anticoagulant therapy. Additionally, clinical presentation, decision to escalate therapy, recanalization, Glasgow Coma Scale, modified Rankin Scale (mRS) and the clinical outcome was assessed. A total of 14 patients ( 9 female patients, 5 male patients), with ages ranging from 17 to 57 years, met the criteria of endovascular therapy. The clinical symptoms of 12 patients had improved after receiving endovascular therapy and only 2 patients suffered from intracranial hemorrhage following the procedure. Complete recanalization of venous sinus was exhibited in 10/14 (71.4\%) patients in group A when compared
\end{abstract}

Correspondence to: Professor Qiujing Wang, Department of Neurosurgery, Shenzhen Hospital of Southern Medical University, 1333 Xinhu Road, Shenzhen, Guangdong 518000, P.R. China

E-mail: edison99x@163.com

${ }^{*}$ Contributed equally

Key words: cerebral venous sinus thrombosis, treatment, stent retriever, thrombectomy, thrombolytic with $1 / 15(6.7 \%)$ patients in group B. mRS were improved in the 12-month follow-up in groups A and B when compared with that at admission. In the present study, patients with acute CVST treated with stent retriever thrombectomy combined with local thrombolytic therapy had a favorable outcome. To conclude, the present study provides a treatment option in treating CVST, particularly for patients that present with evident cortical venous outflow stasis or deteriorate neurology, despite appropriate anticoagulant therapy.

\section{Introduction}

Cerebral venous sinus thrombosis (CVST) is a potentially life-threatening disease due to cerebral venous thrombosis or sinus thrombosis. Approximately 3 to 4 cases of CVST per million adults and 7 cases of CVST per million neonates occur annually $(1,2)$. CVST is typically associated with pregnancy, neoplasm, systemic diseases, puerperium, dehydration, oral contraceptives and coagulopathies (3). However, in $30 \%$ of cases, no underlying etiology can be identified (3). Timely detection of CVST is vital for patients as prompt management prevent fatalities and also averts further disabilities (2). The confirmed diagnosis of CVST relies on the demonstration of thrombus in the sinuses and/or cortical veins by neuroimaging (4). Computed tomography (CT) is useful to rule out tumor or subdural hematoma, particularly for patients suspected of CVST or presenting with subacute headache (4). Direct signs of CVST include the cord sign, the dense triangle sign and the empty delta sign which may be identified in $\sim 1 / 3$ of cases by CT (5). Magnetic resonance venography (MRV) is currently the best noninvasive method to confirm the diagnosis of CVST (6). An abnormal signal in a sinus and the corresponding absence of flow on MRV supports the diagnosis of CVST (7). Compared with MRV, digital subtraction angiography (DSA) not only indicates the thrombosis in sinuses perfectly, but also displays the occlusion in cortical veins precisely, thus this method has become the gold standard of CVST (8).

Systemic anticoagulation is the first-line treatment and the majority of patients with CVST will respond to systemic 
anticoagulation (9). CVST has a favorable prognosis; however, the condition may be associated with neurologic deterioration, despite reasonable treatment with anticoagulation (10). With novel endovascular techniques and devices developed, direct thrombolysis and mechanical thrombectomy without a craniotomy is becoming increasingly general $(7,11-13)$. Endovascular treatment of CVST has the potential advantages of direct dissolution or asportation of clots, allowing recanalization of blood flow, decreased intracranial pressure and rapid improvement of severe symptoms (9).

Recently, mechanical thrombectomy was used as an aggressive therapeutic method for the treatment of CVST, particularly for patients exhibiting clinical deterioration (14). Stent retrievers to treat CVST have previously been indicated in a small sample report (15). However, the therapeutic effect of these devices requires further verification with an abundant number of samples. Local thrombolytic therapy is able to effectively recanalize the thrombosed cortical veins (16). Therefore, individual treatment of mechanical thrombectomy combined with local thrombolytic therapy may achieve an improved curative effect.

In the present study, the medical records of 29 patients with CVST were retrospectively reviewed. From these patients, 14 patients were successfully treated with stent retriever thrombectomy combined with local thrombolytic therapy. The present study identified that, despite anticoagulant treatment failure, the majority of CVST patients with evident cortical venous outflow stasis, rapid worsening of consciousness or neurological deficits had a favorable prognosis following treatment with stent retriever thrombectomy combined with local thrombolytic infusion.

\section{Materials and methods}

Patients. Before the study, ethical approval was received from the Ethics Committee of Zhujiang Hospital (Guangzhou, China) and patients and (or) relatives were informed about the risks and benefits of the procedure and written consent was obtained. We initiated a retrospective on 29 patients (17 women:12 men) who were diagnosed with CVST by magnetic resonance venography (MRV) or digital subtraction angiography (DSA) in Zhujiang Hospital, Southern Medical University, Guangzhou, China between January 2011 and November 2015. The following information was recorded: Age, symptoms, etiological factors, onset, Glasgow Coma Scale (GCS) applied as described by Okamura et al (17), location of the thrombus, recanalization, outcome and complications. Systemic anticoagulation was the initial treatment for all patients following admission. Endovascular treatment was administered to patients presenting with: i) Anticoagulation therapy failure; ii) rapid worsening of consciousness or neurological deficits (GCS $\leq 10$ ); and iii) evident cortical venous outflow stasis (arteriovenous circulation time $>11 \mathrm{sec}$; venous phase $>5 \mathrm{sec}$ ). The following exclusion criteria for receiving endovascular treatment was followed: i) Clinical symptoms were controlled when regular anticoagulant treatment was initiated; ii) no obvious cortical venous outflow stasis was present (arteriovenous circulation time $<11 \mathrm{sec}$; venous phase $<5 \mathrm{sec}$ ). In the present study, 14 patients (group A) received endovascular treatment whereas 15 patients (group B) were given systemic anticoagulant therapy.

Treatment. Patients with CVST in group A underwent stent retriever thrombectomy combined with continued partial thrombolysis. A 6-Fr sheath was placed into the right common femoral artery to allow a diagnostic cerebral angiogram. Subsequently, a 6-Fr guiding catheter was selectively placed in the internal jugular vein through the left femoral vein. A 0.027-inch microcatheter (Rebar-27; eV3 Neurovascular, Irvine, CA, USA) was introduced via the internal jugular vein into the position of the thrombus. A microwire was used to break down thrombi and facilitate the advancement of the microcatheter. Subsequently, the microwire was withdrawn and a 6x30 mm stent (Solitaire AB; eV3 Neurovascular) was semi-deployed at the distal part of the thrombus via the microcatheter. After $5 \mathrm{sec}$, the stent, which covered the clot, and the microcatheter were slowly pulled into the guiding catheter and removed from the body. This procedure was performed 3 to 6 times to retrieve the clot until recanalization of the venous sinus was achieved. Subsequently, the stent was removed from the body and the microcatheter was retained in the superior sagittal sinus for continued partial thrombolysis. Urokinase was infused into the thrombosis site using microinjection techniques at the speed of $5.0 \mathrm{U} / \mathrm{min}$ for 3 to 5 days. The total concentration of urokinase did not exceed 1,000,000 units. Throughout the procedure, the activated clotting time was maintained at 2 to 3 times above the normal value ( 80 to $160 \mathrm{sec}$ ). When a patient presented with venous sinus stenosis, a 6x30 mm stent (Solitaire AB, Medtronic, Inc., Minneapolis, MN, USA) was advanced and deployed at the site of stenosis for angioplasty in order to dilate the venous sinus.

Following admission, group B patients received systemic anticoagulation. During the first 3 days, low molecular weight heparin was infused intravenously at 2,500 to $3,000 \mathrm{IU} / \mathrm{d}$. From the fourth day following treatment, $5 \mathrm{mg} /$ day warfarin was administered orally for 3 to 12 months. The international normalized ratio (INR) was carefully monitored 2 to 4 times in the first two weeks and once in the following four weeks, while patients received anticoagulant therapy.

Follow-up. Follow-up visits were performed at 3, 6 and 12 months via outpatient clinics or over the telephone. MRV or DSA were performed to monitor whether the thrombus recurred in the sinus. Prognosis of patients with CVST was classified according to the modified Ranking Scale (mRS): 0, normal; 1, no significant disability; 2, slight disability (look after own affairs without assistance); 3, moderate disability (required help, able to walk assisted); 4, moderately severe disability (unable to walk assisted); 5 , severe disability (unable to ambulate, altered mentation); and 6, mortality.

Statistical analysis. Data are presented as the mean \pm standard deviation. Groups were compared using the Student's t-test or a one-way analysis of variance, followed by the least significant difference test for pairwise comparisons. Discrete data were given as counts and percentages. $\mathrm{P}<0.05$ was considered to indicate a statistically significant difference. All calculations were evaluated using the SPSS 20.0 statistical package (IBM SPSS, Armonk, NY, USA). 


\section{Results}

Baseline characteristics. From 29 patients, the mean age was 34 years (range, 17-60 years). A total of 17 patients $(58.6 \%$ ) were women and 12 patients $(41.4 \%)$ were men. A total of 16 patients $(55.2 \%)$ exhibited acute onset (within 7 days), 4 patients $(13.8 \%)$ were included into sub-acute onset (7 to 14 days) and 9 patients $(31.0 \%)$ were included into chronic onset ( $>14$ days). Common risk factors of CVST were identified in 15 patients $(51.7 \%)$, puerperium in 7 patients $(24.1 \%)$, head injury in 2 patients $(6.9 \%), 4$ patients $(17.2 \%)$ presented with oral contraceptives and 1 patient (3.4\%) had vasculitis disease. No risk factors were identified in 5 patients $(48.3 \%$; Tables I and II). The predominant clinical presentations were headache, epilepsy, neurologic deficits, nausea, emesis, dizziness, pyrexia and papilledema. The condition of 7/29 patients (24.1\%) deteriorated following admission. Superior sagittal sinus (alone or in combination with other venous sinus) was the primary thrombosis-affected sinus. The affected sinus of each case is summarized in Tables I and II, which was evaluated by DSA or MRV.

Outcome. In the present study, $14 / 29$ patients $(48.3 \%)$ received endovascular therapy whereas $15 / 29$ patients $(51.7 \%)$ received systemic anticoagulant treatment. Additionally, in group A, 2/14 patients (14.3\%) underwent stent-assisted angioplasty. Following the procedure, in group A, the clinical symptoms in 12 patients $(85.7 \%)$ had improved immediately; however, 2 patients $(6.9 \%)$ suffered from intracranial hemorrhage. In group B, the clinical symptoms in $14 / 15$ patients $(93.3 \%)$ had improved; however, 1 patient $(6.7 \%)$ suffered from herniation.

A 38-year-old male with no identified underlying etiology presented with epilepsy and hemiparesis. The patient was admitted to the hospital in May 2011 and was diagnosed with CVST by MRV. Anticoagulation Treatment with heparin was initiated for 3 days; however, there was no improvement in his epilepsy and hemiparesis. DSA revealed thrombose of the superior sagittal sinus, right transverse sinus and right sigmoid sinus. Subsequently, stent retriever thrombectomy combined with local thrombolytic therapy was performed on the patient (Fig. 1). The symptoms subsequently resolved in the following days and the patient was discharged 15 days following admission. The patient presented with no symptoms and there was no recurrence of CVST at the 12-month follow-up.

A 24-year-old male presented with epilepsy for 3 days was admitted to the hospital in June 2012. The patient was diagnosed with CVST and right frontal lobe hemorrhage accompanied with ischemia by MRV and CT. DSA revealed thrombosis occurred in the superior sagittal sinus and left transverse sinus (Fig. 2). Endovascular treatment was subsequently performed on the patient. His symptom was relieved with no thrombosis; however, recurrence of thrombosis in the sinuses at the 12-month follow-up was indicated.

A 55-year-old male presented, with a chief complaint of headache and hemiparesis for 4 days, was admitted to the hospital in October 2013. A CT scan revealed right frontal lobe intracranial hemorrhage and ischemia. Meanwhile, MRV demonstrated the thrombosis in the superior sagittal sinus, left transverse sinus and sigmoid sinus. Stent retriever thrombectomy was performed and urokinase was infused into the thrombosis site using microinjection techniques at the speed of $5.0 \mathrm{U} / \mathrm{min}$ for 3 days (Fig. 3). The patient continued to take oral anticoagulants for 12 months, and there was no recurrence of headaches or hemiparesis at the 12-month follow-up.

A 23-year-old female, who was in puerperium, presented with acute onset of severe headaches, vomiting and generalized seizures. She was immediately treated with systemic anticoagulation therapy for 2 days. However, the patient continued to experience neurologic deficits and fell into a mild coma the following day. Stent retriever thrombectomy combined with local thrombolytic therapy was therefore undertaken. At 3 months, the involved sinuses were completely recanalized and no clinical symptoms were observed. Another 24-year-old female deteriorated on the first postoperative day and intracranial hemorrhage was diagnosed. Endovascular treatment was immediately stopped and anticoagulation therapy with oral warfarin was administered two weeks later. One month later, neurologic deficits were notably improved and the patient was discharged.

A-17-year-old male presented to the hospital with epilepsy and hemiparesis for 15 days. MRI indicated left frontal lobe intracranial hemorrhage and DSA revealed that thrombus occurred in the superior sagittal sinus, left transverse sinus and sigmoid sinus but without cortical venous flow stasis. $\mathrm{He}$ received systemic anticoagulant therapy during the stay of hospital. His symptoms were relieved and he was subsequently discharged after receiving treatment of low molecular weight heparin that was intravenously infused at 3,000 IU/day for the first 3 days and administered orally with warfarin at $5 \mathrm{mg}$ /day for the next 27 days. MRV indicated no obvious recanalization in sinuses and he presented with no symptoms on the 12-month follow-up (Fig. 4).

Recanalization of the venous sinus is demonstrated in Tables I and II. In group A, complete venous flow restoration was obtained in $10 / 14$ patients $(71.4 \%)$, whereas partial venous flow restoration was exhibited in $4 / 14$ patients $(28.6 \%)$ following therapy. Angioplasty was performed in $2 / 14$ patients $(14.3 \%)$ who suffered from sinus stenosis; $11 / 14$ patients (78.6\%) resulted in a positive neurological outcome, whereas residual neurological deficits persisted in 3/14 patients (21.4\%). In group B, $1 / 15$ patients $(6.7 \%)$ obtained complete venous flow restoration, whereas $14 / 15$ patients exhibited partial venous flow restoration following treatment. A total of $11 / 15$ patients presented with no symptoms and 4/15 patients exhibited residual neurological deficits following treatment. Neurological function was evaluated by $\mathrm{mRS}$. The outcome at the different time points is demonstrated in Fig. 5. In group A, stent retriever thrombectomy combined with local thrombolytic infusion significantly improved mRS (at 3 months, 2.79 \pm 0.43 ; at 6 months, 2.07 \pm 0.47 ; and at 12 months, $1.36 \pm 0.50)$ when compared with $\mathrm{mRS}$ prior to treatment $(4.64 \pm 0.50 ; \mathrm{P}<0.05$; Fig. $5 \mathrm{~A})$. In group $\mathrm{B}, \mathrm{mRS}$ (at 3 months, $2.13 \pm 0.83$; at 6 months, $1.33 \pm 0.82$; and at 12 months, $0.87 \pm 0.99$ ) was also significantly improved by systemic anticoagulation when compared with the mRS prior to treatment $(3.54 \pm 0.74 ; \mathrm{P}<0.05$; Fig. 5A). The average GCS increased significantly following endovascular treatment (group A) when compared with that on admission $(\mathrm{P}<0.05$; Fig. 5B). However, there was no obvious improvement in the average GCS following anticoagulant therapy (group B). 


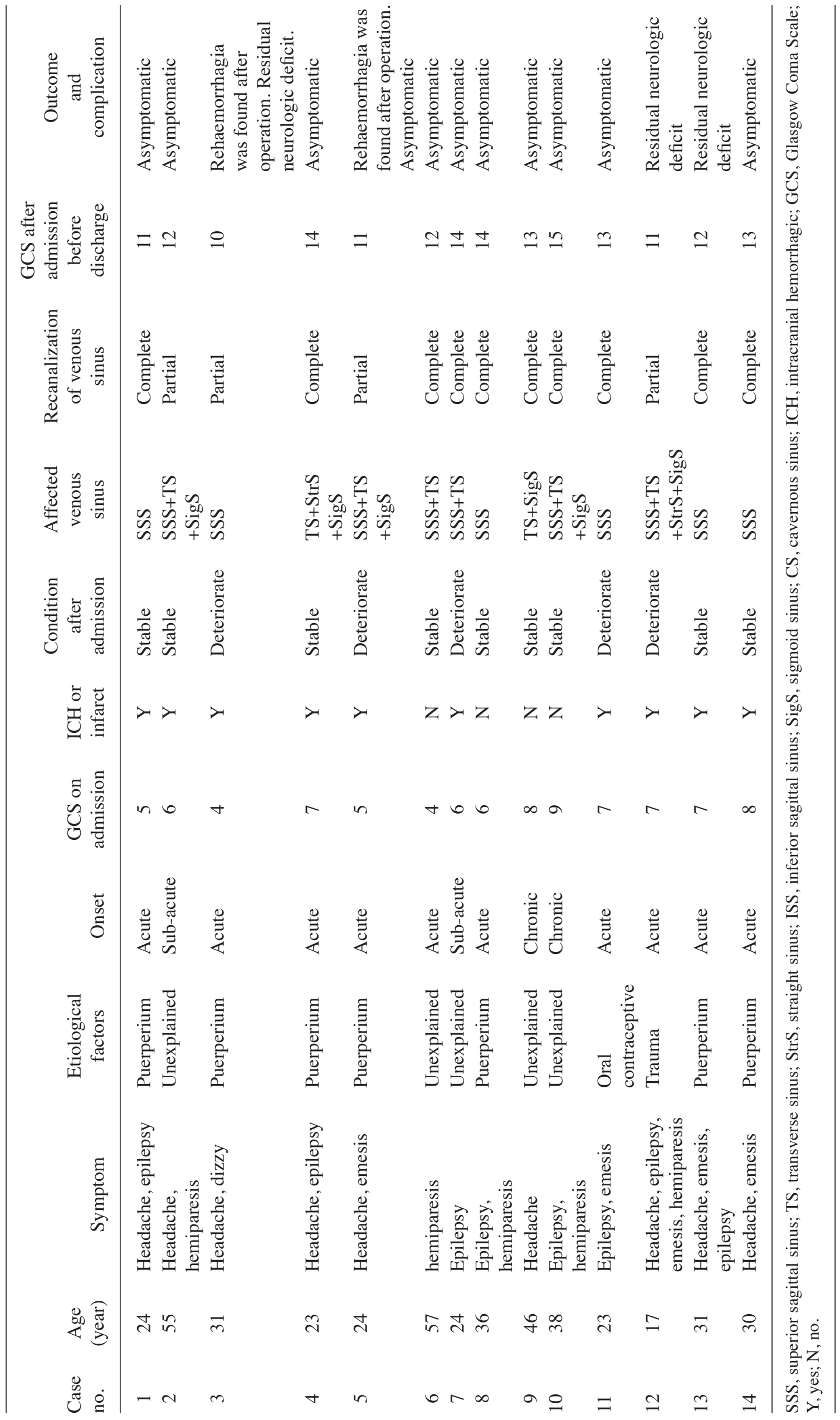




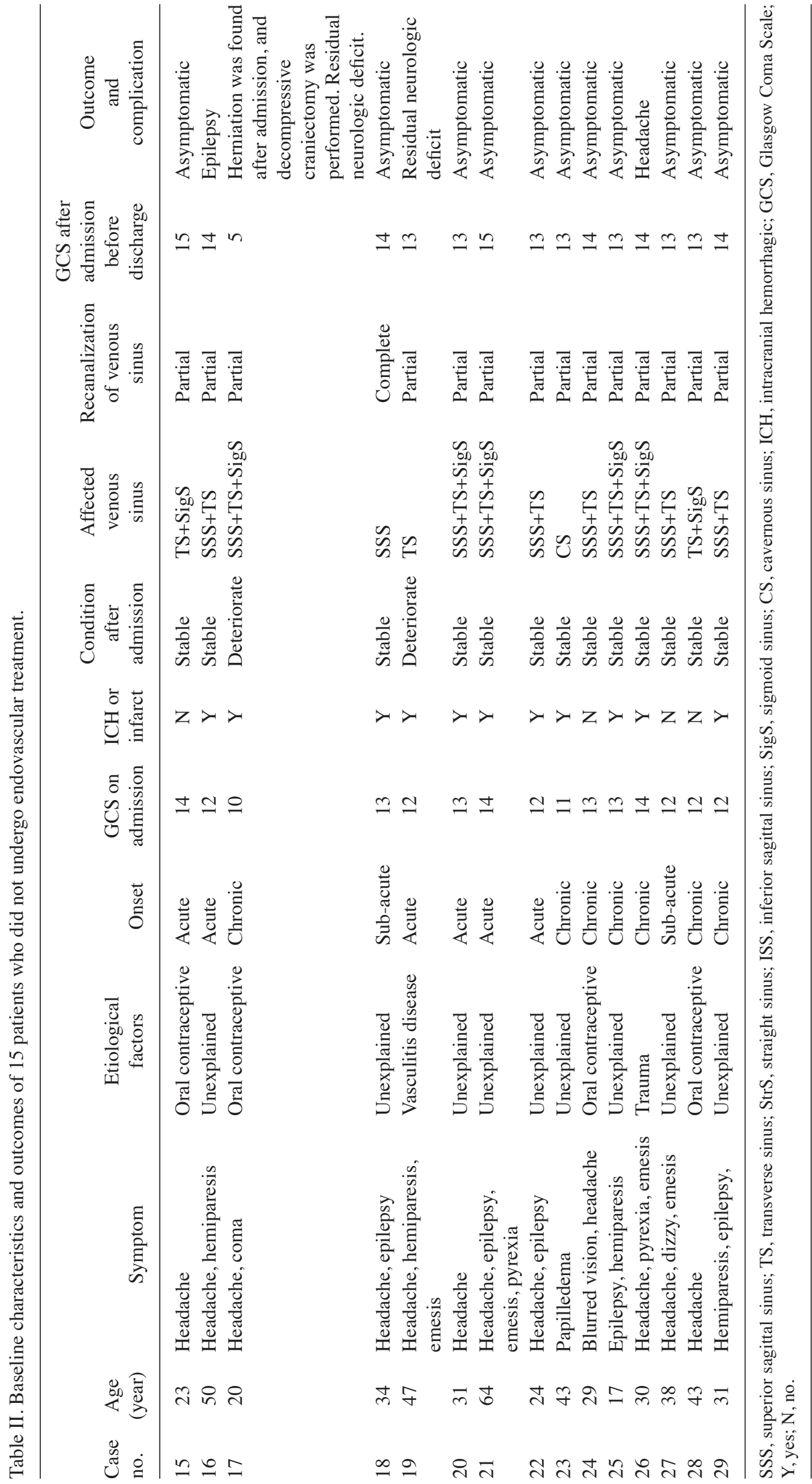




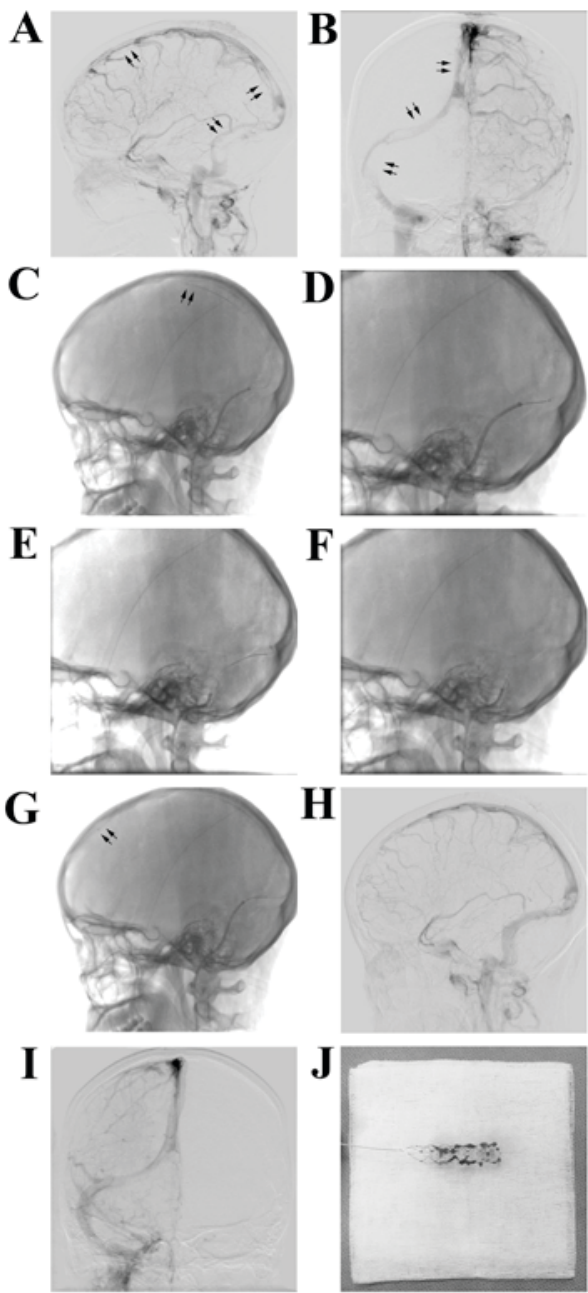

Figure 1. Representative images of a 38-year-old male who received stent retriever thrombectomy combined with local thrombolytic therapy after anticoagulation failure. (A and B) Lateral and posteroanterior DSA images revealed features of extensive cerebral venous sinus thrombosis involving superior sagittal sinus, transverse sinus, sigmoid sinus and significant collateral venous drainage (arrows). (C) A solitaire AB stent was placed into the superior sagittal sinus for mechanical thrombectomy (arrows). (D) The stent and the clots were withdrawn slowly into the microcatheter from the superior sagittal sinus. (E) Another solitaire AB stent was advanced into the transverse sinus and sigmoid sinus for mechanical thrombectomy. (F) Thrombus was removed from the transverse sinus and sigmoid sinus into the microcatheter (G) The microcatheter was indwelled into the superior sagittal sinus for local thrombolytic administration (arrows). (H) Lateral image of DSA revealed recanalization of the superior sagittal sinus following 3-day local urokinase administration. (I) Posteroanterior image of DSA showed recanalization of the transverse sinus and sigmoid sinus. $(\mathrm{J})$ The solitaire AB stent was covered with fresh clots. DSA, digital subtraction angiography.

\section{Discussion}

Patients with CVST may present with a variety of symptoms, including headaches, hemiparesis, seizures, sensory disturbances, and other focal deficits (18); however, headaches are the most common clinical symptom $(4,19)$. In the present study, intracerebral hemorrhage infarct, which is typically associated with a severe clinical presentation at onset and results in a poor outcome (20), occurred in $\sim 2 / 3$ of patients with CVST.

There was a female predominance in CVST in the present study, which is in agreement with previous findings $(3,21)$. Common risk factors for CVST include: Hypercoagulable disorders due to, for example, oral contraceptive use, puerperium, or hematological malignancies; head trauma; intracranial hypotension; and systemic infections (3). In the present study, 12 patients were considered to be in a hypercoagulable state. A total of 7 patients were in puerperium and 4 patients were using oral contraception. In a previous report, underlying etiology could not be identified in $30 \%$ of patients who involved with CVST (3). In the present study, no known risk factor was identified in $12 / 29$ patients (41.4\%), which was higher than the previous report (3).

Treating CVST with mechanical thrombectomy has been reported previously (14). Although no relevant randomized trials have been performed, data from previous cases have revealed that mechanical thrombectomy has notable advantages as a treatment approach for CVST and promotes a high recanalization rate and favorable prognosis $(4,12,14,15,22,23)$. Therefore, mechanical thrombectomy has been the first-line tool for neurologists in treating patients with worsening clinical deterioration following anticoagulant therapy.

A series of different mechanical endovascular treatments have been reported previously. These include rheolytic thrombectomy, the Penumbra system, the Merci device, Solitaire AB or FR $(4,12,14,15,22,23)$. The AngioJet system is the most commonly used rheolytic thrombectomy device. In a retrospective study by Dashti et al (24), 13 patients with CVST were treated with rheolytic thrombectomy and thrombosed intracranial sinuses were recanalized in all patients following treatment. Although the AngioJet device has an advantage in recanalizing the occluded sinuses immediately due to the application of the Bernoulli principle (25), it is hard to deliver the device to the target sinuses due to stiffness of the delivery system (26). Therefore, the AngioJet system may not be an optimal option in treating thrombus in tortuous sinuses.

The Penumbra system allows for continuous aspiration of the thrombotic fragments the fragment of thrombus. In the meantime, the sinus wall is protected by a separator. The flexible delivery system allows the device to reach the thrombosed sinuses effortlessly. Choulakian and Alexander (27) reported that 4 adult patients with CVST who underwent mechanical thrombectomy using the 0.041 -inch Penumbra system achieved satisfactory sinus patency. Furthermore, Velat et al (28) reported the successful treatment of female treated with direct thrombectomy using 0.054-inch Penumbra thromboaspiration catheter. The patient improved symptomatically following the intervention and no thrombus recurred at the 6-month follow-up.

The Merci clot retriever is a snare-type device that removes thrombus using a corkscrew-shaped wire (11). Repeatedly pulling and pushing of the microsnare in the clot can increase the surface of the thrombolytic agents; however, this also increases the risk of endothelium damage (29). The Merci device offers a prompt resolution and can be used in combination with other mechanical thrombectomy methods or thrombolytic agents (30). Khan et al (11) successfully used the device in combination with local recombinant tissue plasminogen activator infusion to restore flow in the superior sagittal sinus of a middle-aged woman.

Mechanical thrombectomy approaches have been utilized individually or in various combinations (31). For example, in some cases, balloon angioplasty combined with mechanical thrombectomy is a sufficient alternative for treating CVST with 


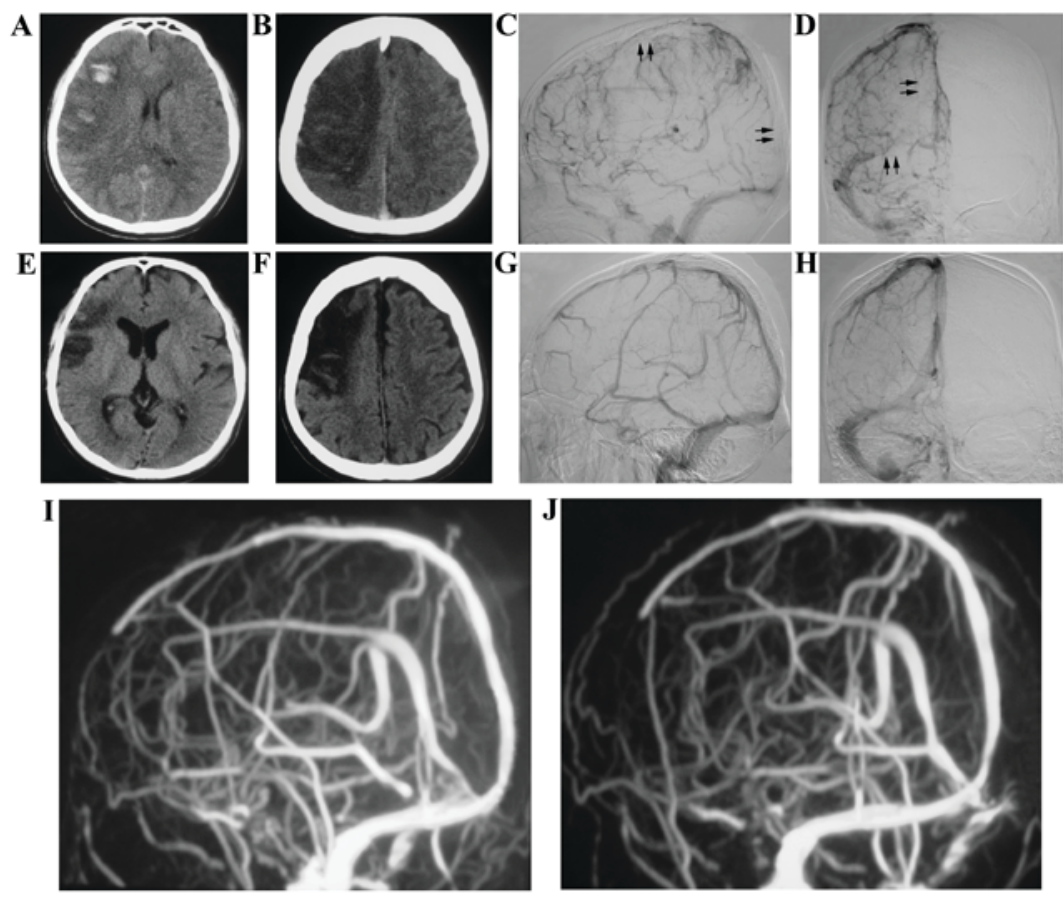

Figure 2. A 24-year-old male received stent retriever thrombectomy combined with local thrombolytic therapy following admission. (A and B) A CT scan showed the wide range of hemorrhagic lesions and ischemia in the right frontal lobe before treatment. (C and D) DSA images revealed that thrombosis occurred in the overall length of the superior sagittal sinus and left transverse sinus (arrows). (E and F) Intracerebral hemorrhage had been absorbed, meanwhile, ischemic infarction was decreased at the 3-month follow-up. (G and H) DSA indicated the recanalization of the superior sagittal sinus and left transverse sinus at the 3-month follow-up. MRV showed no thrombosis recurred in sinuses at (I) 6-month and (J) 12-month follow-up. DSA, digital subtraction angiography; MRV, magnetic resonance venography; CT, computed tomography.

sinus stenosis (7). Stent retrievers (solitaire AB or FR), approved by the Food and Drug Administration for the treatment of ischemic stroke caused by large vessel occlusion, have become the first-line tools for endovascular treatment of stroke (32). In a previous case report, Froehler was the first to utilize a large-sized stent retriever $(6 \times 30 \mathrm{~mm})$ to treat CVST (5). The self-expanding design of stent retrievers provide flexibility and the device is able to be effectively deployed in the thrombosis sigmoid (15). Later case reports have indicated promising treatment results using stent retrievers $(22,32)$; however, due to the limited sample size it is difficult to draw any conclusion.

In the present study, within the 29 cases, stent retriever thrombectomy combined with local thrombolytic therapy was performed in 14 cases. In a previous study, in order to dissolve intravascular thrombus, rapidly metabolized thrombolytic agents, such as urokinase and recombinant tissue plasminogen activator (rt-PA), were delivered to the thrombosis site using a microcatheter (31). A number of studies claim that local thrombolytic therapy is reasonably safe for acute deteriorating patients $(9,33)$; however, its efficacy cannot be assessed from the available data due to the lack of randomized controlled trials. Furthermore, local thrombolytic therapy, which may increase the local drug concentration in the thrombosis sinus and reduce the total dosage of thrombolytic agents when compared with systemic thrombolysis $(34,35)$, may lower the risk of hemorrhage (13). Thrombosis in cortical veins has been indicated to be a critical factor in the development of irreversible brain tissue damage in animal models of CVST $(36,37)$. Moreover, evident cortical venous outflow stasis results in the increased risk of hemorrhagic infarct and brain edema (38).
Hence, recanalization in thrombosed cortical veins is a key factor to rescue brain tissue damage. Furthermore, unobstructed cortical venous drainage is able to reduce the risk of hemorrhage (39). Thrombolytic agents are able to contact the surface of the thrombus in cortical veins that mechanical techniques cannot reach and prevent vessel angiolysis (31). Therefore, the present study suggested that mechanical thrombectomy in combination with local continuous infusion of urokinase provides promising recanalization rates in sinuses and cortical veins.

CVST likely leads to an increase in venous pressure of the sinus and cortical veins, which causes venous congestion that results in hemorrhagic infarction and localized vasogenic edema (40). Furthermore, venous congestion likely reduces arterial perfusion, causing ischemia, which leads to neuronal cell death (41). Thrombolytic therapy may continue for several days and may increase the risk of hemorrhagic complications, particularly in patients with pre-existing hemorrhagic venous infarction. However, those who present with clinical worsening may still benefit from intravascular thrombolytic therapy $(9,33,42)$. In the present study, 10/14 (71.4\%) patients with pre-existing hemorrhage received local continuous infusion of urokinase following stent retriever thrombectomy. Only 2/10 (20\%) patients experienced intracranial hemorrhage once intrasinus thrombolytic therapy was initated. A young female suffered from intracranial hemorrhage two days following intrasinus thrombolytic therapy and fell into a deep coma. Her symptoms were relieved following decompressive craniectomy and the patient gradually recovered over the course of three months. 


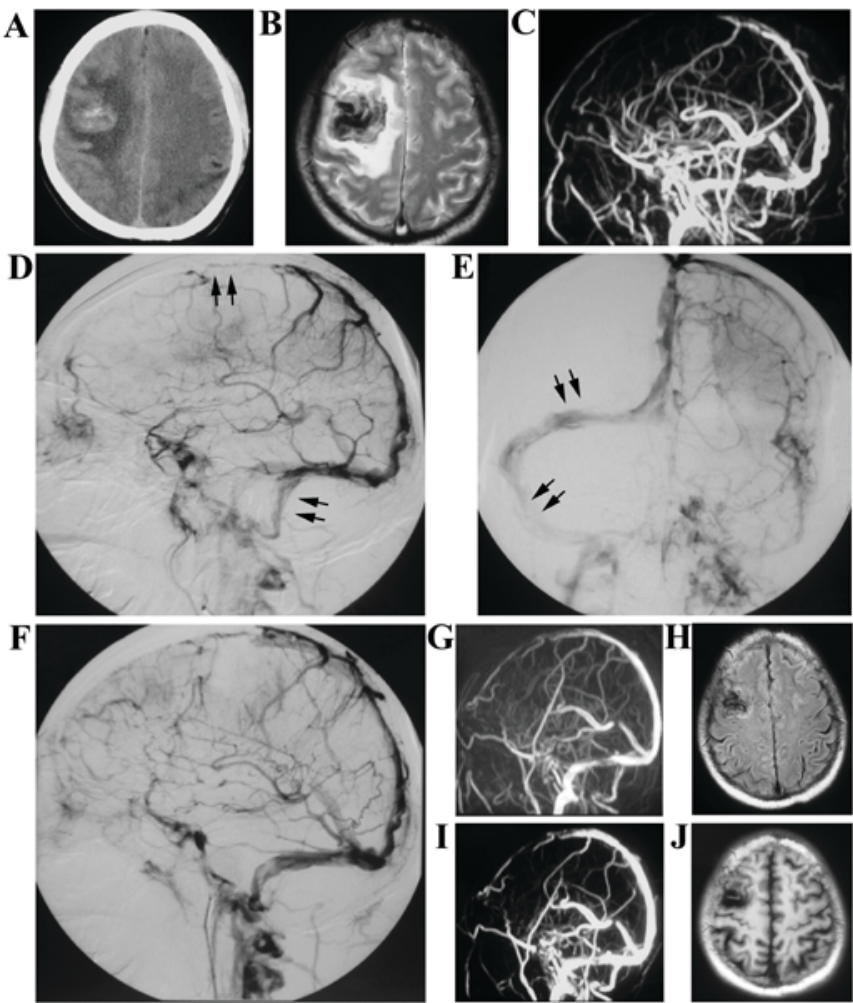

Figure 3. Representative images of a 55-year-old male who received endovascular treatment after being admitted to hospital. (A) A CT scan and (B) MRI imaging revealed right frontal lobe intracranial hemorrhage and ischemia prior to treatment. (C) MRV indicated complete occlusion of the posterior one half of the superior sagittal sinus, left transverse sinus and sigmoid sinus. (D and E) DSA images revealed the occlusion of the posterior part of superior sagittal sinus, left transverse sinus and sigmoid sinus (arrows). (F) DSA imaging showed the partial recanalization of sinuses following stent retriever thrombectomy and local thrombolytic therapy with urokinase. At the 3-month follow-up, (G) MRV imaging revealed the patency of the superior sagittal sinus and left sigmoid sinus, and reduced residual thrombus in the left transverse sinus. (H) MRI revealed that the hematoma in right frontal lobe was absorbed and the ischemic infarction was also decreased. (I) Follow-up MRV imaging at 12 months demonstrated complete recanalization in the superior sagittal sinus and left sigmoid sinus, and partial recanalization in the left transverse sinus. (J) MRI indicated the old infarction in the frontal lobe. MRI, magnetic resonance imaging; DSA, digital subtraction angiography; MRV, magnetic resonance venography; CT, computed tomography.

CVST patients with mild symptoms, GCS $\geq 10$ and fluent cortical venous drainage, received systemic anticoagulant treatment at the time of hospitalization. Only 1 patient achieved complete recanalization of the venous sinus; however, the majority of patients had a favorable prognosis, despite the low recanalization rate of venous sinus. This may be explained by the compensation of cortical venous flow and neuronal function restoration, which has been demonstrated in a novel CVST animal model (43). Therefore, systemic anticoagulant therapy is still an optimal option for treating CVST patients who present with superior neurological status.

CVST has been demonstrated to have a good prognosis, dependent on early diagnosis and prompt anticoagulant therapy treatment (2). In the present study, the majority of CVST patients that presented with evident cortical venous outflow stasis, rapid worsening of consciousness or neurological deficits treated with stent retriever thrombectomy combined with local thrombolytic infusion had a favorable prognosis. Dosages
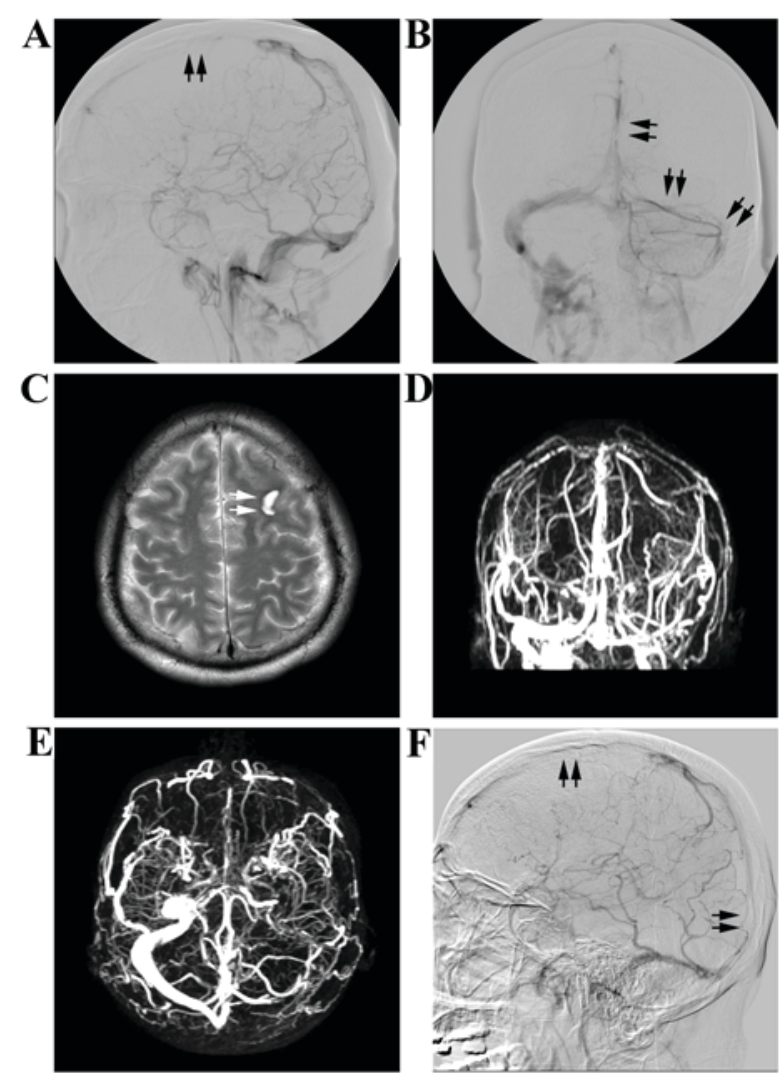

Figure 4. A-17-year-old male with mild symptoms received systemic anticoagulant therapy following admission. (A and B) DSA revealed that thrombus occurred in the anterior half of the superior sagittal sinus, left transverse sinus and sigmoid sinus (arrows). However, no obvious cortical venous flow stasis was observed. (C) MRI indicated left frontal lobe intracranial hemorrhage on admission (arrows). (D and E) At the 3-month follow-up, MRV showed no obvious recanalization of the sagittal sinus, left transverse sinus and left sigmoid sinus. (F) DSA revealed no obvious change in the thrombosed sinuses at the 12-month follow-up (arrows). However, the patient presented with no symptoms on follow-up. MRI, magnetic resonance imaging; DSA, digital subtraction angiography; MRV, magnetic resonance venography.

of the thrombolytic agents used range from single bolus injections to continuous infusion $(9,31,33)$. In the present study, we indicated that prolonged infusion with a small dosage of thrombolytic agents may establish an adequate recanalization rate and may also avoid the recurrence of hemorrhage. MRV and DSA are the optimal approaches used for confirming the patency of the venous sinuses and cerebral veins, and subsequently determining recanalization $(20,21)$. In the present study, oral anticoagulant treatment with warfarin effectively avoided the recurrence of thrombus when evaluated by MRV or DSA at follow-ups.

In conclusion, the present findings suggest that favorable outcomes may be achieved using stent retriever thrombectomy combined with local thrombolytic therapy in patients with CVST who exhibit cortical venous outflow stasis or severe neurological deterioration, despite systemic anticoagulation. However, despite the favorable therapeutic benefits, the risk of hemorrhagic complications may be an important end point during the treatment process. Therefore, large scale randomized controlled trials are required to establish and compare mechanical thrombectomy alone to mechanical thrombectomy in combination with local thrombolytic therapy in the future. 
A

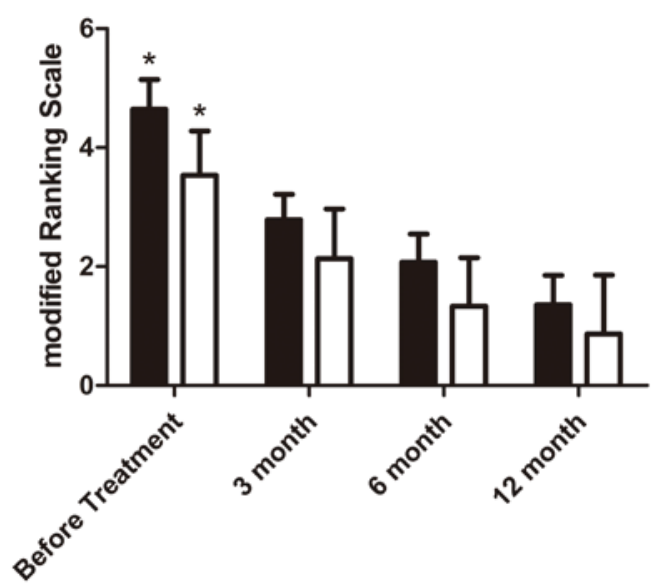

B

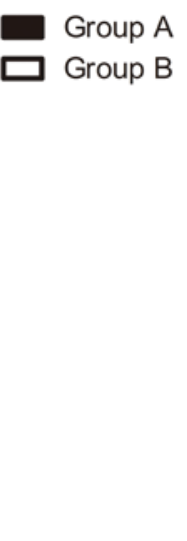

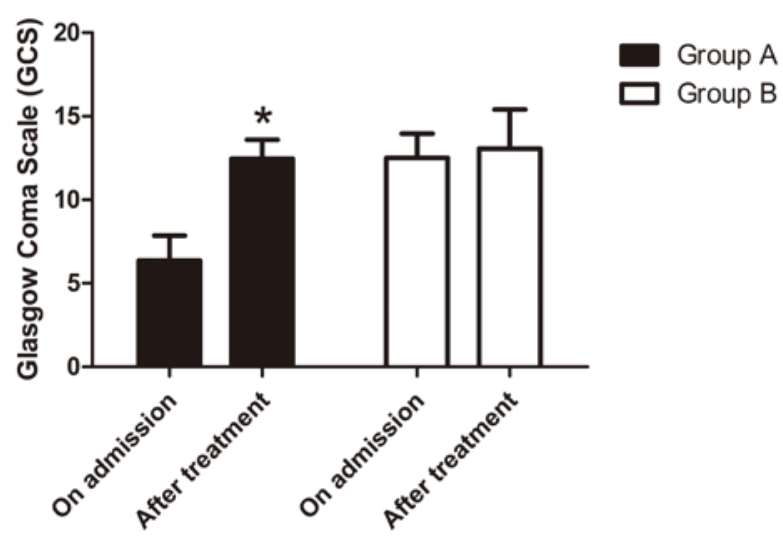

Figure 5. (A) mRS at different time points. $\mathrm{mRS}$ at 3, 6 and 12 months were significantly improved when compared with pre-treatment in both two groups. "P<0.05 vs. 3, 6 and 12 months. (B) GCS revealed that in group A ( $\mathrm{n}=14)$, GCS improved significantly following endovascular treatment when compared with that on admission. However, there is no obvious changes of GCS in group B ( $n=15)$. "P<0.05 vs. on admission. Group A, patients received stent retriever thrombectomy combined with local thrombolytic therapy; Group B, received systemic anticoagulant treatment. mRS, modified Rankin Scale; GCS, Glasgow Coma Scale.

\section{Acknowledgements}

The Guangdong Provincial Department of Science and technology provided financial support in the form of Guangdong Provincial Clinical Medical Centre for Neurosurgery funding (grant no. 2013B020400005) and the Special Program of Guangdong Province, China (grant no. 00136530154773042). Haizhuqu District Department of Science and Technology provided financial support in the form of Science and Technology Program of Haizhu District, Guangzhou, Guangdong Province, China (grant no. 2013-cg-28). The sponsors had no role in the design or conduct of this research.

\section{References}

1. Martinelli I, Bucciarelli P, Passamonti SM, Battaglioli T, Previtali E and Mannucci PM: Long-term evaluation of the risk of recurrence after cerebral sinus-venous thrombosis. Circulation 121: 2740-2746, 2010.

2. Ferro JM,Canhão $P, S t a m J, B o u s s e r M G$ and BarinagarrementeriaF and ISCVT Investigators: Prognosis of cerebral vein and dural sinus thrombosis: Results of the international study on cerebral vein and dural sinus thrombosis (ISCVT). Stroke 35: 664-670, 2004.

3. Saadatnia M, Fatehi F, Basiri K, Mousavi SA and Mehr GK: Cerebral venous sinus thrombosis risk factors. Int J Stroke 4: 111-123, 2009.

4. Ferro JM and Canhão P: Cerebral venous sinus thrombosis: Update on diagnosis and management. Curr Cardiol Rep 16: 523, 2014.

5. Buonanno FS, Moody DM, Ball MR and Laster DW: Computed cranial tomographic findings in cerebral sinovenous occlusion. J Comput Assist Tomogr 2: 281-290, 1978.

6. Klingebiel R, Bauknecht HC, Bohner G, Kirsch R, Berger J and Masuhr F: Comparative evaluation of 2D time-of-flight and 3D elliptic centric contrast-enhanced MR venography in patients with presumptive cerebral venous and sinus thrombosis. Eur J Neurol 14: 139-143, 2007.

7. Chow K, Gobin YP, Saver J, Kidwell C, Dong P and Viñuela F: Endovascular treatment of dural sinus thrombosis with rheolytic thrombectomy and intra-arterial thrombolysis. Stroke 31: 1420-1425, 2000.
8. Qu H and Yang M: Early imaging characteristics of 62 cases of cerebral venous sinus thrombosis. Exp Ther Med 5: 233-236, 2013.

9. Rahman M, Velat GJ, Hoh BL and Mocco J: Direct thrombolysis for cerebral venous sinus thrombosis. Neurosurg Focus 27: E7, 2009.

10. Mortimer AM, Bradley MD, O'Leary S and Renowden SA: Endovascular treatment of children with cerebral venous sinus thrombosis : A case series. Pediatr Neurol 49: 305-312, 2013.

11. Khan SH, Adeoye O, Abruzzo TA, Shutter LA and Ringer AJ: Intracranial dural sinus thrombosis: Novel use of a mechanical thrombectomy catheter and review of management strategies. Clin Med Res 7: 157-165, 2009.

12. Raychev R, Tateshima S, Rastogi S, Balgude A, Yafeh B, Saver JL, Vespa PM, Buitrago M and Duckwiler G: Successful treatment of extensive cerebral venous sinus thrombosis using a combined approach with Penumbra aspiration system and Solitaire FR retrieval device. BMJ Case Rep 2013: 1-5, 2013.

13. Yue X, Xi G, Zhou Z, Xu G and Liu X: Combined intraarterial and intravenous thrombolysis for severe cerebral venous sinus thrombosis. J Thromb Thrombolysis 29: 361-367, 2010.

14. Siddiqui FM, Dandapat S, Banerjee C, Zuurbier SM, Johnson M, Stam J and Coutinho JM: Mechanical thrombectomy in cerebral venous thrombosis: Systematic review of 185 cases. Stroke 46: 1263-1268, 2015.

15. Froehler MT: Successful treatment of cerebral venous sinus thrombosis with the Solitaire FR thrombectomy device. J Neurointerv Surg 5: e45, 2013.

16. Wasay M, Bakshi R, Kojan S, Bobustuc G, Dubey N and Unwin DH: Nonrandomized comparison of local urokinase thrombolysis versus systemic heparin anticoagulation for superior sagittal sinus thrombosis. Stroke 32: 2310-2317, 2001.

17. Okamura K: Glasgow coma scale flow chart: A beginner's guide. Br J Nurs 23: 1068-1073, 2014.

18. Wang JW, Li JP, Song YL, Tan K, Wang Y, Li T, Guo P, Li X, Wang Y and Zhao QH: Clinical characteristics of cerebral venous sinus thrombosis. Neurosciences (Riyadh) 20: 292-295, 2015.

19. Alberti A, Venti M and Biagini S: Headache and cerebral vein and sinus thrombosis. Front Neurol Neurosci 23: 89-95, 2008.

20. Stam J: Thrombosis of the cerebral veins and sinuses. N Engl J Med 352: 1791-1798, 2005.

21. Ferro J, Lopes M and Rosas M: Long-term prognosis of cerebral vein and dural sinus thrombosis. Stroke 35: 664-670, 2004.

22. Shaikh H, Pukenas BA, Mcintosh A, Licht D and Hurst RW: Combined use of solitaire FR and penumbra devices for endovascular treatment of cerebral venous sinus thrombosis in a child. J Neurointerv Surg 7: e10, 2015. 
23. Nimjee SM, Powers CJ, Kolls BJ, Smith T, Britz GW and Zomorodi AR: Endovascular treatment of venous sinus thrombosis: A case report and review of the literature. J Neurointerv Surg 3: 30-33, 2011.

24. Dashti SR, Hu YC, Yao T, Fiorella D, Mitha AP, Albuquerque FC and McDougall CG: Mechanical thrombectomy as first-line treatment for venous sinus thrombosis: Technical considerations and preliminary results using the AngioJet device. J Neurointerv Surg 5: 49-53, 2013.

25. Borhani Haghighi A, Mahmoodi M, Edgell RC, Cruz-Flores S, Ghanaati H, Jamshidi M and Zaidat OO: Mechanical thrombectomy for cerebral venous sinus thrombosis: A comprehensive literature review. Clin Appl Thromb Hemost 20: 507-515, 2014

26. Zhang A, Collinson RL, Hurst RW and Weigele JB: Rheolytic thrombectomy for cerebral sinus thrombosis. Neurocrit Care 9 : $17-26,2008$

27. Choulakian A and Alexander MJ: Mechanical thrombectomy with the penumbra system for treatment of venous sinus thrombosis. J Neurointerv Surg 2: 153-156, 2010.

28. Velat GJ, Skowlund CJ, Waters MF, Mocco J and Hoh BL: Direct thrombectomy using the Penumbra thromboaspiration catheter for the treatment of cerebral venous sinus thrombosis. World Neurosurg 77: 591.e15-18, 2012.

29. Kirsch J, Rasmussen PA, Masaryk TJ, Perl J II and Fiorella D: Adjunctive rheolytic thrombectomy for central venous sinus thrombosis: Technical case report. Neurosurgery 60: E577-E578, 2007.

30. Philips MF, Bagley LJ, Sinson GP, Raps EC, Galetta SL, Zager EL and Hurst RW: Endovascular thrombolysis for symptomatic cerebral venous thrombosis. J Neurosurg 90: 65-71, 1999.

31. Qiu Z, Sang H, Dai Q and Xu G: Endovascular treatments for cerebral venous sinus thrombosis. J Thromb Thrombolysis 40: 353-62, 2015.

32. Mokin M, Lopes DK, Binning MJ, Veznedaroglu E, Liebman KM, Arthur AS, Doss VT, Levy EI and Siddiqui AH: Endovascular treatment of cerebral venous thrombosis: Contemporary multicenter experience. Interv Neuroradiol 21: 520-526, 2015.

33. Kamal AK: Thrombolytic therapy in cerebral venous sinus thrombosis. J Pak Med Assoc 56: 538-40, 2006.
34. Li G, Zeng X, Hussain M, Meng R, Liu Y, Yuan K, Sikharam C, Ding Y, Ling F and Ji X: Safety and validity of mechanical thrombectomy and thrombolysis on severe cerebral venous sinus thrombosis. Neurosurgery 72: 730-738, 2013.

35. Bagley LJ, Hurst RW, Galetta S, Teener J and Sinson GP: Use of a microsnare to aid direct thrombolytic therapy of dural sinus thrombosis. AJR Am J Roentgenol 170: 784-786, 1998.

36. Nakase H, Kakizaki T, Miyamoto K, Hiramatsu K and Sakaki T: Use of local cerebral blood flow monitoring to predict brain damage after disturbance to the venous circulation: Cortical vein occlusion model by photochemical dye. Neurosurgery 37 : 285-286, 1995.

37. Fries G, Wallenfang T, Hennen J, Velthaus M, Heimann A, Schild H, Perneczky A and Kempski O: Occlusion of the pig superior sagittal sinus, bridging and cortical veins: Multistep evolution of sinus-vein thrombosis. J Neurosurg 77: 127-133, 1992.

38. Frerichs KU, Deckert M, Kempski O, Schürer L, Einhäupl K and Baethmann A: Cerebral sinus and venous thrombosis in rats induces long-term deficits in brain function and morphology-evidence for a cytotoxic genesis. J Cereb Blood Flow Metab 14: 289-300, 1994.

39. Akins PT, Axelrod YK, Ji C, Ciporen JN, Arshad ST, Hawk MW and Guppy KH: Cerebral venous sinus thrombosis complicated by subdural hematomas: Case series and literature review. Surg Neurol Int 4: 85, 2013

40. Ito K, Tsugane R, Ikeda A, Suzuki Y and Sato K: Cerebral hemodynamics and histological changes following acute cerebral venous occlusion in cats. Tokai J Exp Clin Med 22: 83-93, 1997.

41. Singh R, Cope WP, Zhou Z, De Witt ME, Boockvar JA and Tsiouris AJ: Isolated cortical vein thrombosis: Case series. J Neurosurg: 427-433, 2015.

42. Christo PP, Carvalho GM and Gomes Neto AP: Cerebral venous thrombosis: Study of fifteen cases and review of literature. Rev Assoc Med Bras 56: 288-292, 2010.

43. Chen C, Wang Q, Gao Y, Lu Z, Cui X, Zheng T, Liu Y, Li X, He X, Zhang X, et al: Photothrombosis combined with thrombin injection establishes a rat model of cerebral venous sinus thrombosis. Neuroscience 306: 39-49, 2015. 